\title{
Correction to: Adrift across tectonic plates: molecular phylogenetics supports the ancient Laurasian origin of old limnic crangonyctid amphipods
}

\author{
Denis Copilaş-Ciocianu ${ }^{1,2}$ (D) $\cdot$ Dmitry Sidorov $^{3} \cdot$ Andrey Gontcharov $^{3}$
}

Published online: 30 April 2019

(C) Gesellschaft für Biologische Systematik 2019

\section{Correction to: Organisms Diversity \& Evolution https://doi.org/10.1007/s13127-019-00401-7}

The publisher regrets that the original version of this article contained an error.

Figures 1 and 2 were shown in the wrong order. The original article has been corrected.

Publisher's note Springer Nature remains neutral with regard to jurisdictional claims in published maps and institutional affiliations.

The online version of the original article can be found at https://doi.org/ 10.1007/s13127-019-00401-7

Denis Copilaş-Ciocianu denis.copilas-ciocianu@gamtc.lt

1 Laboratory of Evolutionary Ecology of Hydrobionts, Nature Research Centre, Akademijos 2, 08412 Vilnius, Lithuania

2 Department of Ecology, Faculty of Science, Charles University, Viničná 7, 12844 Prague, Czechia

3 Federal Scientific Centre of the East Asia Terrestrial Biodiversity, Far Eastern Branch of the Russian Academy of Sciences, 100-let Vladivostoku Av. 159, Vladivostok 690022, Russian Federation 